\title{
INCUlCATING SAFETy MANAgEMENT THROUgh AN ELECTRONIC LEARNING MODULE
}

\author{
Jeffrey S. Castrucci and Graeme W. Norval P.Eng. \\ Minerva Safety Management Education Inc. and \\ Department of Chemical Engineering and Applied Chemistry, University of Toronto \\ email: jeffrey.castrucci@mail.utoronto.ca and graeme.norval@utoronto.ca
}

\begin{abstract}
Inculcating a safety focused mindset is an important part of an engineering education, as highlighted by the Canadian Engineering Accreditation Board curriculum requirement to cover occupational health and safety. Despite the importance of covering safety related material, many university faculty are hesitant to cover elements of safety management due to unfamiliarity with the material. In response, Minerva Safety Management Education has developed teaching module slide sets covering a wide range of safety management topics that are freely available for instructors to use in their classes.
\end{abstract}

In this paper, we report on the next phase of module deployment: the transformation of a slide set into an electronically facilitated, self-contained, independent learning module. This new format includes narrated lessons and automatic grading of comprehension tests. The format removes the time burden from instructors and teaching assistants, while enabling students to absorb the material at their own pace outside of class instructional hours. The module is designed to be compatible with existing learning management systems and easily incorporated into existing course websites. We report student feedback on this new format and share findings applicable to the development and conversion of future self-contained electronic learning modules.

Keywords: Engineering Education; Safety Culture; Online Learning.

\section{INTRODUCTION}

\subsection{Motivation}

Inculcating a safety focused mindset is an important part of an engineering education, as highlighted by the Canadian Engineering Accreditation Board curriculum requirement to cover occupational health and safety [3]. Despite the importance of covering safety related material, many university faculty are hesitant to cover elements of safety management due to unfamiliarity with the material. In response, Minerva Safety Management Education Inc. has developed teaching module slide sets covering a wide range of safety management topics that are freely available for instructors to use in their classes [10]. Minerva Safety Management Education Inc. is a non-profit health and safety advocacy group which focuses on influencing post-secondary education curriculum in the areas of engineering and business [11].

\subsection{Literature Review}

Safety management is acknowledged as a range of thinking and cultural approaches that benefits from multidisciplinary perspectives when taught to engineering students $[7,8]$. Safety culture in academic laboratories has been a subject of recent focus in response to a number of tragedies [1,5], and student engagement in building a safety culture in academic laboratories is an early opportunities to prepare students to be leaders of safety culture in whatever organizations the find themselves in during their careers $[2,4]$. However, laboratory safety is not the only element of workplace health and safety that students need to be informed about. An understanding of risk terminology and concepts is beneficial to safety management in both academic and industrial workplace settings. Due to its wide applicability, a Minerva module on risk management was selected as the first module to be converted into a self-contained electronic learning module.

\subsection{Module Content and Goals}

Minerva Safety Management Education Inc. has previously identified the development of curriculum resources for safety related teaching as an area of significant opportunity for impact [6] resulting in the development of twenty-four slide deck modules now freely available [10]. The next goal is the conversion of these slide deck modules into self-contained learning modules compatible with existing course management 
software. A module on Risk Management was selected as the first to be converted, and we report lessons learned from this experience.

The slide module was converted into a series of eight videos, each between five and ten minutes in length, divided into six submodules. Dividing the module into smaller pieces was identified as a desirable trait to allow students to digest content in smaller to manage pieces and to be able to return to where they left off without needing to hunt through a 90 minute video. Each video showed the corresponding slides in sequence and was narrated. Videos were recorded with TechSmith Relay screen capture and recording software [12] and were edited with Freemaker Video Convert editing software [9] into a final form of MP4 video files with a resolution of 720 x 1280. For the initial testing, the videos were hosted on the University of Toronto media server.

At the end of each module was a quiz with two to three questions of either single answer or multiple answer multiple choice questions. These quiz questions covered content from the just completed module. The entire module takes between one and one half and two hours to complete.

The module was constructed in the Blackboard course management software, which is already in use for all course at the University of Toronto. The module can be exported into a number of different formats compatible with different learning management systems, and is available on the Minerva website. This was done to make the content as easily available to users as possible, regardless of the learning management system in use at their institution.

\subsection{Structure for Evaluation}

The module was tested with a third year chemical engineering design course of 140 students at the University of Toronto. Participation was voluntary and no marks were associated with interacting with the content. In addition to the content described in Section 1.3, for this initial test of the content, two surveys, one to be completed before beginning the module and one to be completed after completing the module were included. These survey questions were intended to gauge students' attitudes toward safety content in general and the module in particular before and after the module, and in what contexts the students accessed the module content. The before and after module survey questions are included in Appendix A.

\section{RESULTS AND DISCUSSION}

\subsection{Response Rate}

Of the class of 140, 23 students complete at least one quiz, 14 students completed all six quizzes, 7 students answered the before module survey, and 3 students answered the after module survey. Further details on number of respondents are shown in Fig. 1. All the hypotheses intended to be tested with the before and after module surveys were intended to compare before survey responses to after survey responses, so the lack of after survey responses was a major obstacle to evaluating the efficacy of the module. While the feedback will be useful for improving the modules, the feedback is of insufficient magnitude for meaningful statistical hypothesis testing.

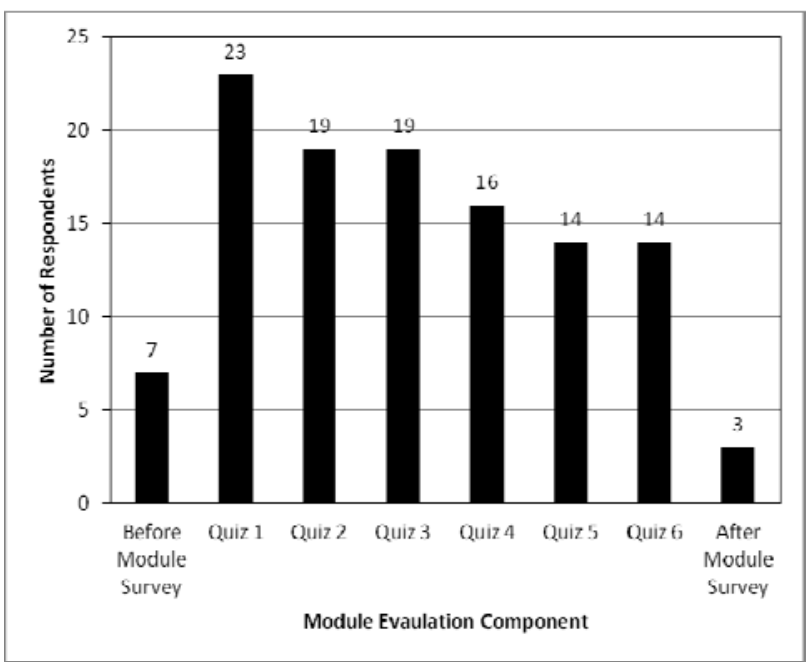

Fig. 1. Number of student respondents to each module evaluation component.

If we were to do module testing again, more effort should be devoted to encouraging student participation, perhaps by assigning a small mark value to completion the module.

\subsection{Quiz Responses}

Quizzes were computer marked questions covering content in each of the six submodules. Questions which tested the understanding of the difference between probability of an accident and consequence of an accident based upon an incident description were only correctly answered at a rate of $50 \%$, indicating this is a continuing area of confusion. Other questions were primarily answered correctly (> $90 \%$ correct for most questions) except those with multiple correct answers ( 70 \% correct), where written feedback highlighted the presence of some ambiguously worded questions that have been 
modified before the general release. The high correct response rates could be the result of a number of effects including insufficiently challenging questions and may support the unstructured feedback suggestion that "more in-depth" quiz questions could be added to improve the challenge induced engagement of the learning experience.

\subsection{Survey Responses}

\subsubsection{Students' affective support of safety} management ideas. Questions were asked before and after the module to test changes in belief about the importance of risk management to engineering practice.

Questions designed to gauge affective buy-in to the concept that safety management thinking is valuable knowledge for an engineer were all strongly agreed with in both the before and after module surveys, so no change in sentiment could be detected. The three after module survey participants were divided on whether they would recommend the module to a colleagues as a useful learning experience. Given the responses to the affect testing survey questions, it is likely the only participants were those in the class who already strongly support the ideology of safety management.

2.3.2 Students' perception of their prior exposure to risk management learning. Questions were asked before and after the module to test changes in perception of previous safety content based upon exposure to new safety content.

Questions designed to gauge previous expose to safety content indicated that third year students believe they had already been exposed to material relating to risk management, in particular near unanimous strongly agree responses to having learned about risk management before and learning the technical definitions of risk and hazard before. As best as can be observed from the limited after module sample size, these positions were unchanged after the module was completed. Further, responses indicate that the material covered was not new to a second semester third year engineering student, so conclusions about change in sentiment achieved by the module could not be drawn. Given the module content would be suitable for first year engineering or business students, it is unsurprising that third year students felt the content was not new.

2.3.3 How students interacted with the content. Questions asked after the module to assess how the students accessed and interacted with the content.

Questions designed to determine method of access (stationary computer/laptop/mobile phone) and how the module was approached (sequential watching of videos alternating with corresponding quizzes vs. watching all videos and then doing all quizzes vs. doing quizzes and simultaneously hunting in videos to find answers) indicated a range of access devices and approaches used among the respondents. This suggests the open ended structure to the module is an asset to student learning as each student can customize their learning experience by experiencing the content in their preferred order.

2.3.4 Unstructured feedback. Written and verbal feedback obtained after the students completed the module about how they viewed the experience and what they thought could have been done differently.

Feedback was strongly supportive of the structure of 5-10 minute video sizes, the ability to restart where they left off, and the ability to access the videos and quizzes in any order. There was an interest in being able to watch the videos at a faster speed, which the UofT media server system does not give as an option. As such, the final version of the videos has been made available on YouTube. There was a common theme that the quiz questions could have been more clearly worded and would have benefitted from asking "more in-depth" questions. The quizzes being set to report the correct answer in the event of an incorrect answer was also identified as a desirable trait that was lacking. This feature was enabled before general release of the module.

\section{CONCLUSIONS}

While the number of responses from students given the opportunity to complete the module were fewer than hoped for, which impeded or ability to measure any inculcation effects of the module, quiz responses and before and after module surveys did yield some feedback that allowed improvement of the module. In particular, changes which clarified and improved the formative aspects of quiz questions were implemented and videos were made more accessible through hosting on YouTube. The conversion of future module slide decks to this selfcontained learning module format has been demonstrated to be feasible, and the lessons learned could likely also be applied to other Internet based learning environments, such as massive open online courses (MOOCs).

\section{Acknowledgements}

We acknowledge Minerva Safety Management Education for funding to develop the learning module. We acknowledge Donna Cortolezzis and Vic Pakalnis for development of the original slide deck and we thank the engineering students of CHE 334 H1S 2016 for their feedback on the testing of this module.

\section{References}

[1] ACS Joint Board/Council Committee on Chemical Safety, Creating Safety Cultures in Academic Institutions. 
Washington, DC: The American Chemical Society (ACS), 2012, 63 pp.

Available as of May 3, 2016 from http://www.acs.org/content/dam/acsorg/about/governance/c ommittees/chemicalsafety/academic-safety-culture-reportfinal-v2.pdf

[2] Shayna Burchett, et al., "Exploding misconceptions: Developing a culture of safety through learner driven activities," J. Chem. Health Safety, http://dx.doi.org/10.1016/j.jchas.2016.04.001, 2016.

[3] Engineers Canada, Core Engineering Competencies. Ottawa, ON: Canadian Engineering Accreditation Board (CEAB), 2012, 8 pp.

Available as of May 3, 2016 from

https://www.engineerscanada.ca/sites/default/files/w_Compete ncies_and_Feedback.pdf

[4] Kathryn A. McGarry, et al., "Student Involvement in Improving the Culture of Safety in Academic Laboratories," J. Chem. Edu., vol. 90, pp. 1414-1417, 2013.

[5] Gary L. Messing, "Creating a safety culture in academic laboratories,” MRS Bull., vol. 38, pp. 592-593, August 2013.

[6] Graeme Norval, Vic Pakalnis, and Tony Pasteris, “Teaching Occupational Health and Safety in Engineering Schools Best Practices, Support, and Opportunities," in Proc. CEEA Canadian Engineering Education Conf., CEEC10, Andy Fisher (ed.) (Kingston, ON; 7-9 June 2010), 7 pp., 2010.

[7] Joseph H. Saleh and Cynthia C. Pendley, "From learning from accidents to teaching about accident causation and prevention: Multidisciplinary education and safety literacy for all engineering students,” Reliability Eng. Sys. Safety, vol. 99, pp. 105-113, 2012.

[8] Joseph H. Saleh, Karen B. Marais, and Francesco M. Favaro, "System safety principles: A multidisciplinary engineering perspective,” J. Loss Prev. Proc. Ind., vol. 29, pp. 283-294, 2014.

[9] http://www.freemake.com/free_video_converter/

[10] http://safetymanagementeducation.com/teachingresources/teaching-modules/

[11] http://safetymanagementeducation.com/

[12] https://www.techsmith.com/techsmith-relay.html

\section{APPENDIX A: BEFORE \& AFTER MODULE SURVEYS}

\section{A.1 Before Module Survey}

\section{Preamble:}

This survey is completely voluntary. Completing this survey will not impact your mark in this course in any way. Your replies will be anonymous and will not be linked to your individual identity. The responses will be used by the teaching staff to improve the course experience for future students.

Please complete this survey before watching any of the module videos or completing any of the module quizzes.

\section{Consent Question: (True/False)}

This survey is completely voluntary. Completing this survey will not impact your mark in this course in any way. Your replies will be anonymous and will not be linked to your individual identity. The responses will be used by the teaching staff to improve the course experience for future students.

This survey should only be complete before watching any of the videos or attempting any of the quizzes.

I have read and understood the description of the survey and consent to the use of my responses.

\section{Questions: (5 point Likert Scale)}

I have learned about risk management during previous courses of my current undergraduate engineering degree.

I have learned and recall the technical definitions for the terms "risk" and "hazard" during previous courses of my current undergraduate engineering degree.

Regardless of needing to fulfill course requirements, it will be beneficial to my future career goals to learn about workplace safety and risk management.

Workplace safety and risk management is relevant to all workplaces.

Engineers have a positive contribution to make to impact safety in their workplaces.

An engineer's duty to protect public welfare should be their first priority.

\section{A.2 After Module Survey}

\section{Preamble:}

This survey is completely voluntary. Completing this survey will not impact your mark in this course in any way. Your replies will be anonymous and will not be linked to your individual identity. The responses will be used by the teaching staff to improve the course experience for future students.

Please complete this survey after completing the Risk Management Independent Learning Module.

\section{Consent Question: (True/False)}

This survey is completely voluntary. Completing this 
survey will not impact your mark in this course in any way. Your replies will be anonymous and will not be linked to your individual identity. The responses will be used by the teaching staff to improve the course experience for future students.

This survey should only be complete after completing the module.

I have read and understood the description of the survey and consent to the use of my responses.

Questions: (Single Choice)

The best description of how I completed the Risk Management Learning Module is:

watched the videos one at a time and then answered the corresponding quiz questions

watched all the videos, then went back and answered all the quiz questions

did not watch the videos, then answered the quiz questions

watched all the videos, then did not answer the quiz questions

read the quiz questions, then hunted through the videos to find the answers

did not watch the videos or answer the quizzes

another strategy

The best description of how I watched the Risk Management Learning Module videos is:

By myself, with other people who are also my class mates, with other people who are not my class mates, did not watch the videos, other

The best description of how I completed the Risk Management Learning Module quizzes is:

By myself, with some people who are my class mates, with other people who are not my class mates, did not answer the quizzes, other

\section{Questions: (Select All That Apply)}

I viewed the Risk Management Learning Module videos using the following methods (select all that apply):

Desktop or other Stationary Computer, Laptop or Tablet Computer, Mobile Phone or other Handheld Computer, Other

I answered the Risk Management Learning Module quizzes using the following methods (select all that apply):
Desktop or other Stationary Computer, Laptop or Tablet Computer, Mobile Phone or other Handheld Computer, Other

\section{Questions: (Single Choice)}

The method I used most often to view the Risk Management Learning Module videos was:

Desktop or other Stationary Computer, Laptop or Tablet Computer, Mobile Phone or other Handheld Computer, Other

The method I used most often to answer the Risk Management Learning Module quizzes was:

Desktop or other Stationary Computer, Laptop or Tablet Computer, Mobile Phone or other Handheld Computer, Other

Questions: (5 point Likert Scale)

I have learned about risk management during previous courses of my current undergraduate engineering degree.

I have learned and recall the technical definitions for the terms "risk" and "hazard" during previous courses of my current undergraduate engineering degree.

Regardless of needing to fulfill course requirements, it has been beneficial to my future career goals to learn about workplace safety and risk management.

Workplace safety and risk management is relevant to all workplaces.

Engineers have a positive contribution to make to impact safety in their workplaces.

An engineer's duty to protect public welfare should be their first priority.

The content of the Risk Management Learning Module was primarily new material.

I can imagine myself recommending the Risk Management Learning Module to a friend or colleague in the future.

\section{Freeform Writing Textbox:}

Is there anything else you would like to tell us about your experience using the Risk Management Learning Module? For instance, what worked well? What would you have prefer was different? Did we leave anything out of the survey or course content that you think should have been included? Also, if you answered "Other" and can give more detail, or otherwise want clarify your answers to any of the questions, you can do that here. 